\title{
Assessment of Bipolar Radiofrequency Ablation Combined with Coronary Artery Bypass Surgery for Management of Atrial Fibrillation in Cardiac Patients
}

\author{
Hamdy Singab, PhD \\ Department of Cardiovascular and Thoracic Surgery, Faculty of Medicine, Ain Shams University, \\ Ain Shams University Hospitals, Abbasia Square, Cairo, Egypt
}

\section{ABSTRACT}

Background: Atrial fibrillation (AF) is a common problem in patients undergoing coronary artery bypass graft (CABG). For AF ablation, bipolar radiofrequency ablation (BRA) achieves complete transmural ablation lines and reduces the risk of treatment failure. We analyzed the efficacy of BRA for sinus rhythm restoration in patients with $\mathrm{AF}$ undergoing CABG.

Methods: This prospective study included patients with permanent or paroxysmal AF scheduled to undergo BRA combined with CABG in our institution from May 2014 to June 2020. After discharge from hospital, all patients were seen every 6 months over 5 years to evaluate survival, sinus rhythm restoration, and New York Heart Association (NYHA) class.

Results: We enrolled 168 patients, 97 (57.7\%) with permanent AF (group I) and 71 (42.3\%) with paroxysmal AF (group II) at 60 months. We found that group II patients had better sinus rhythm restoration rates after BRA with CABG than group I patients $(P=.005)$. Overall mortality at 60 months was significantly lower in group II patients $(2[2.8 \%])$ than patients in group I $(14[14.4 \%] ; P=.01)$. The survival rate was significantly higher in group II than in group I (94\% versus $72 \% ; P=.0003)$ as shown by KaplanMeier analysis. The $95 \%$ confidence interval of the Cox hazards survival regression ratio was significantly different between groups (0.1792 [0.04069 to 0.7896]; $P=.006)$. Long-term AF (>3 years) before BRA with CABG and permanent AF type were identified as predictors of post-BRA recurrent $\operatorname{AF}(P=.0001$ and $P=.005$, respectively). NYHA class improved significantly at 60 months compared with baseline $(P<.0001)$.

Conclusions: This study identified preoperative AF type and duration as predictors of the success of BRA combined with CABG.

Received August 24, 2020; received in revised form September 5, 2020; accepted September 8, 2020.

Correspondence: Hamdy Singab, PhD, Department of Cardiac Surgery, Faculty of Medicine, Ain Shams University, Abbasia Square, P.O. Box 11517, Cairo, Egypt; 00201001008859; fax: 002022604465 (e-mail: hamdi_singab@ botmail.com, drhamdy-ahmed@med.asu.edu.eg).

\section{INTRODUCTION}

Current estimates of atrial fibrillation (AF) prevalence worldwide suggest that there are $>6$ million patients with AF, and this number is expected to increase to 10 million by 2030 [Gammie 2008; Martin-Suarez 2007]. AF is more frequent in patients undergoing cardiac surgery than in other surgical patients: ischemic heart disease and valvular heart disease are well-known risk factors for AF development [Miyasaka 2006]. A high incidence of ischemic heart disease is found among patients with $\mathrm{AF}(>45 \%)$, whereas $\mathrm{AF}$ incidence is relatively low among patients with proven ischemic heart disease $(<6 \%)$ [Cameron 1988; Otterstad 2006].

Each year in North America, >14,000 AF ablation procedures are combined with cardiac surgery [Gammie 2008]. Recent evidence confirms that postoperative $\mathrm{AF}$ is responsible for significant morbidity and mortality in patients who have undergone coronary artery bypass graft (CABG), including thromboembolism, stroke, heart failure, and adverse reactions to antiarrhythmic drugs [Fuster 2006; Santangeli 2014]. Wyse et al. [2002] demonstrated that medical therapy has limited success in sinus restoration in AF patients. Therefore, catheter ablation is considered to be the first-line therapy, followed by surgical ablation if catheter ablation fails. However, there have been no head-to-head comparisons of postoperative clinical outcomes of catheter ablation and surgical ablation of AF [Starck 2015].

AF waves start from multiple foci at the orifices of the 4 pulmonary veins, and reentrant circuits are responsible for its activation and maintenance [Haissaguerre 1998]. The Cox-Maze III surgical ablation procedure was introduced in 1988. With this method, cuts are made, and atria and pulmonary veins are sutured to create a corridor of scar tissue that directs the electrical impulse from the sinoatrial node to the atrioventricular node [Prasad 2003]. Experience assessed in 1 center up to 2001 indicated that it was the most successful surgical strategy for AF ablation when combined with CABG. However, uptake has been low because of surgical difficulty and high incidence of postoperative complications [Melby 2013]. Efforts have been made to simplify surgical ablation by finding an effective energy source, such as radiofrequency, to achieve complete transmural ablation lines [Benussi 2000; Melo 2000]. Early experience with unipolar radiofrequency technology revealed unpredictable narrow gaps in ablation lines that failed to block AF. By contrast, bipolar radiofrequency ablation (BRA) achieves complete transmural ablation 
lines and improves the likelihood of sinus rhythm restoration in AF patients [Melby 2005; Prasad 2003].

The main purpose of this study is to assess the efficacy of epicardial BRA to achieve sinus rhythm restoration in patients with $\mathrm{AF}$ undergoing CABG. This study is original and is of importance to researchers in the field of cardiac surgery and electrophysiology.

\section{METHODS}

\section{Study Design and Participants}

This was a prospective, nonrandomized, double-armed study performed in 1 study center. Patients with myocardial infarction, left atrium $>65 \mathrm{~mm}$, left ventricular dysfunction, ejection fraction $<30 \%$, or left atrial thrombosis or who needed emergency surgery were excluded from the study. The study was approved by the local ethics committee and conducted in accordance with the Declaration of Helsinki. Each patient gave informed written consent before the operative procedures. The preoperative and demographic characteristics of the patients are given in Table 1 .

\section{Selection Criteria}

The study included all patients who had AF (permanent or paroxysmal) and were scheduled to undergo surgical $\mathrm{AF}$ ablation by BRA combined with CABG in our institution from May 2014 to June 2020. In all, 168 patients underwent CABG combined with BRA. Group I included 97 patients $(57.7 \%)$ who underwent CABG combined with BRA for permanent $\mathrm{AF}$ (longstanding, persistent $\mathrm{AF}$ for $>1$ year). Group II included 71 patients $(42.3 \%)$ who underwent CABG combined with BRA for paroxysmal AF (recurrent AF that terminated spontaneously in $<1$ week) (Figure 1). The mean age of group I was $58 \pm 9$ years, and of group II, $57 \pm 8$ years; both males and females were included.

\section{Epicardial BRA Technique}

BRA was performed using a Cardioblate BP2 device (Medtronic, Minneapolis, MN) (Figure 2). After initiation of the cardiopulmonary bypass, the pericardial reflection was dissected, and the jaws of the bipolar Cardioblate BP2 were clamped around the atrial cuff to isolate the upper right pulmonary veins, lower right pulmonary veins, left pulmonary veins, and left atrial appendage. Right atrial appendage ablation was done at the end of bypass, twice at the same site, with radiofrequency set at $30 \mathrm{~W}$ for 30 seconds each time. Once BRA was complete, CABG was performed. Before the end of cardiopulmonary bypass, an intravenous loading bolus of 300 mg amiodarone (Cardio-Mep; Mepaco-Medifood, Heliopolis, Egypt) was administered, and a slow intravenous infusion of $900 \mathrm{mg} /$ day was started and continued for 24 hours.

\section{Data Collection, Postoperative Ablation Management, and Follow-Up Protocol}

Patients were monitored with continuous ambulatory electrocardiogram (ECG) recording for $7 \pm 2$ postoperative days. If patients developed recurrent $\mathrm{AF}$ during hospitalization,

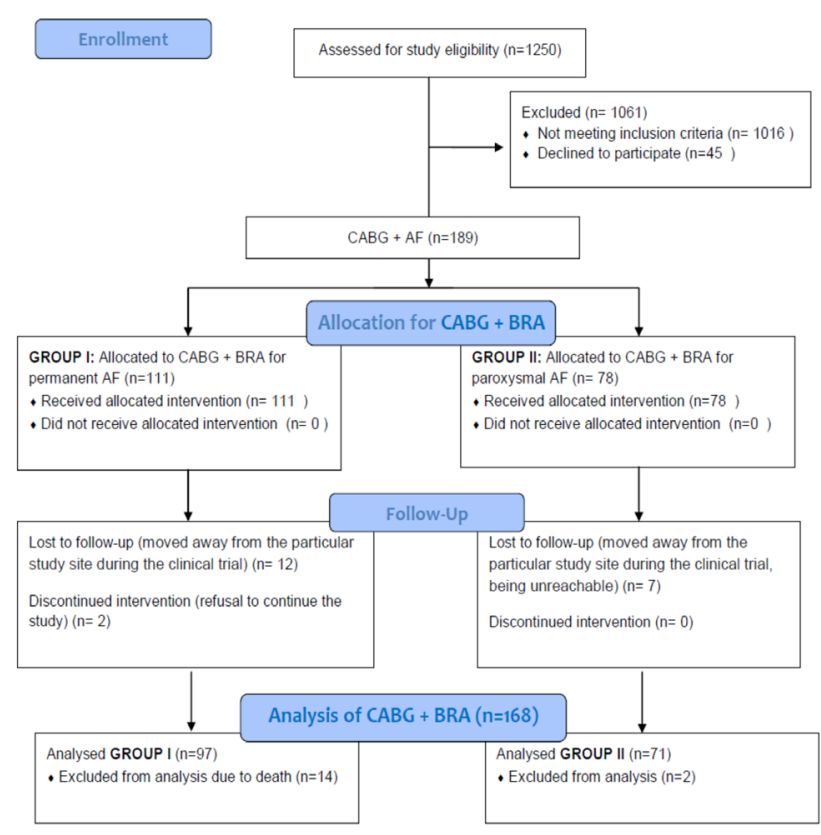

Figure 1. Flow chart of the nonrandomized CABG + BRA trial design.

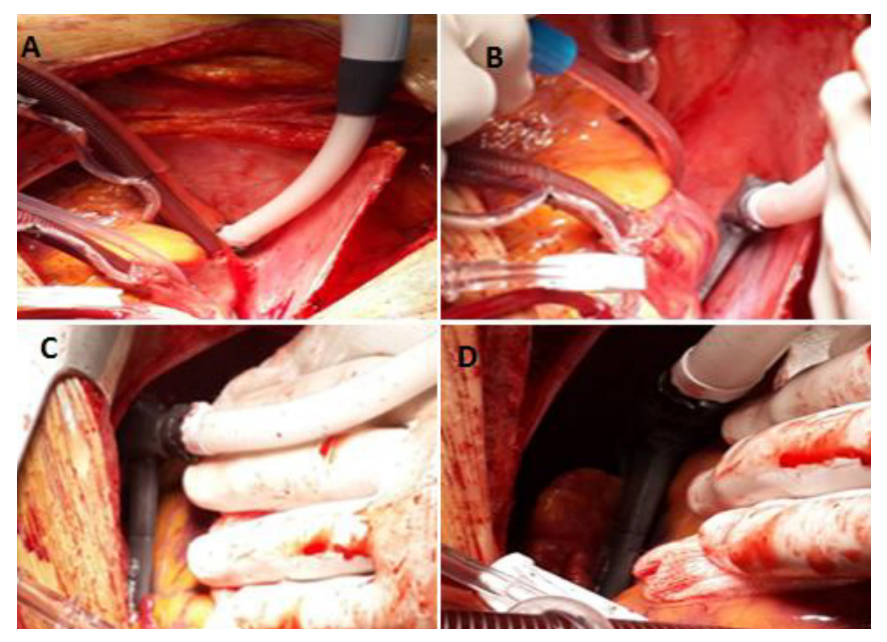

Figure 2. BRA using Cardioblate BP2 device. (A and B) BRA of the right pulmonary veins. ( $C$ and $D$ ) BRA of the left pulmonary veins and left atrial appendage.

amiodarone was started or continued. On the second postoperative day, a maintenance dose of $200 \mathrm{mg}$ amiodarone (Coradrone; Sanofi Aventis, Gentilly, France) once daily orally was prescribed. If amiodarone was contraindicated, $80 \mathrm{mg}$ oral sotalol (Betacore; Amoun Pharmacytical, Cairo, Egypt) twice per day was prescribed. We recommended pacing and antiarrhythmic drug avoidance if patient had bradycardia, atrioventricular block, or nodal rhythm.

Every 6 months over 5 years, all patients were examined at our cardiac surgery and electrophysiology outpatient clinic. After each visit, patients underwent Holter monitoring for 5 days. Recurrent $\mathrm{AF}$ was defined as $\mathrm{AF}>30$ seconds in the first 
Table 1. Preoperative and Demographic Variables*

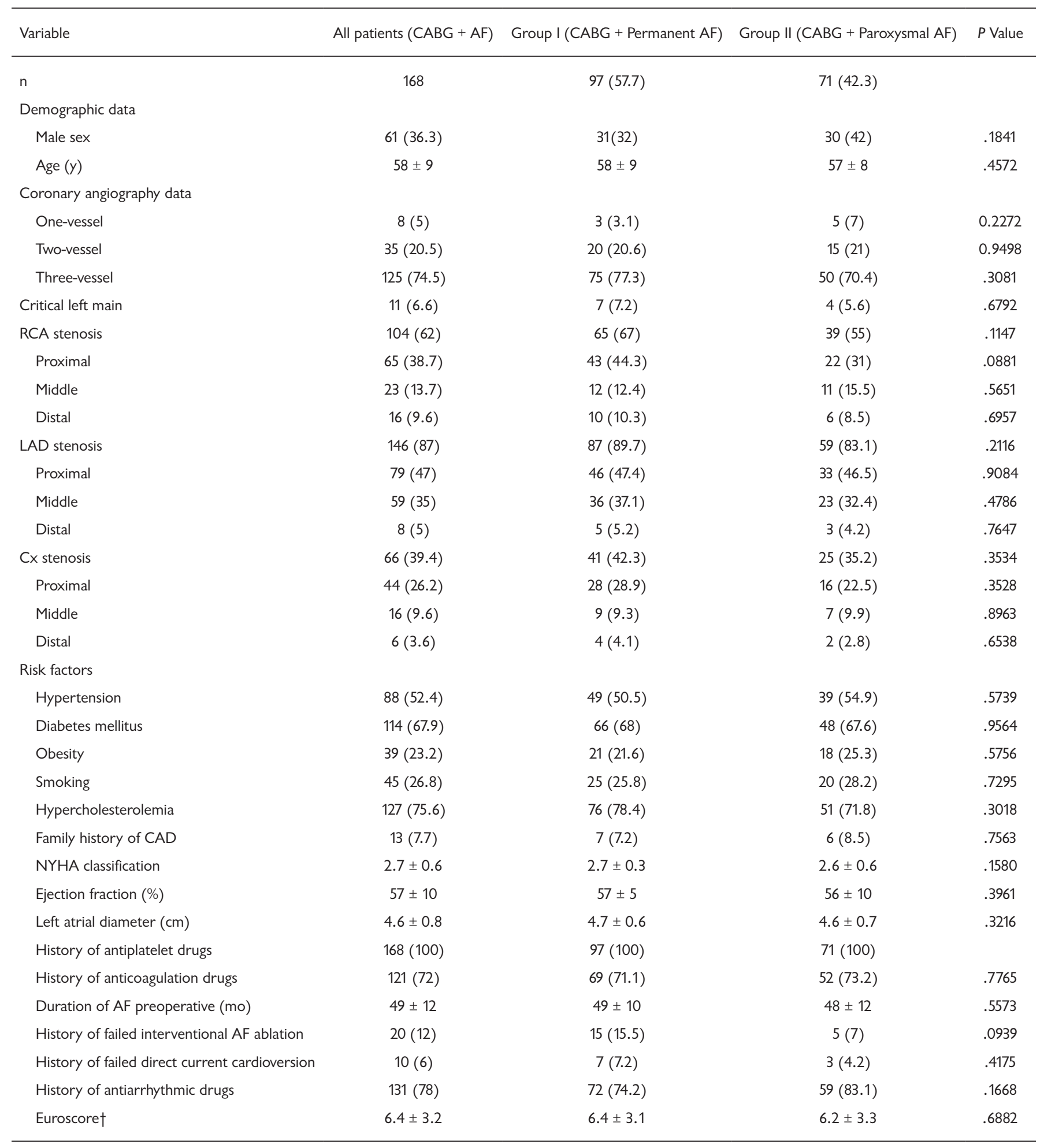

*Data are $\mathrm{n}(\%)$ or mean $\pm \mathrm{SD}$.

†Euroscore predicts risk of cardiac operations (low risk, <2 points; moderate risk, 3 to 5 points; high risk, $>6$ points).

CAD indicates coronary artery disease; Cx, circumflex coronary artery; LAD, left anterior descending coronary artery; RCA, right coronary artery. 
Table 2. Operative Variables of 168 AF Patients Undergoing Bipolar Radiofrequency Ablation Combined with CABG*

\begin{tabular}{lccc}
\hline Variables & All patients CABG + AF & Group I CABG + Permanent AF & Group II CABG + Paroxysmal AF \\
\hline $\mathrm{n}$ & 168 & $97(57.7)$ & $71(42.3)$ \\
CABG (single clamp technique) & $168(100)$ & $97(100)$ & $71(100)$ \\
CPB time (min) & $115.6 \pm 35.2$ & $115.5 \pm 33.2$ & $114.9 \pm 35.2$ \\
ACC time (min) & $75.6 \pm 15.7$ & $75.6 \pm 15.3$ & $75.1 \pm 15.6$ \\
Bipolar AF ablation time (min) & $1.7 \pm 0.6$ & $1.7 \pm 0.5$ & $1.6 \pm 0.9$ \\
Total bipolar ablation time (min) & $12.5 \pm 1.8$ & $12.5 \pm 1.5$ & $12.8 \pm 1.1$ \\
Complete revascularization & $150(89.3)$ & $87(89.7)$ & .9103 \\
Incomplete revascularization & $18(10.7)$ & $10(10.3)$ & .8359 \\
Number of grafts & $3.1 \pm 0.3$ & $3.1 \pm 0.8$ & .3598 \\
Conduits & $168(100)$ & & .1554 \\
LIMA & $8(4.7)$ & $97(100)$ & .8365 \\
RIMA & $66(39.3)$ & $3(3)$ & .8365 \\
Radial artery & $155(92.3)$ & $39(40)$ & .0936 \\
Great saphenous vein & $90(92.8)$ & $71(100)$ \\
\hline
\end{tabular}

*Data are $\mathrm{n}(\%)$ or mean \pm SD.

ACC indicates aortic cross-clamp; CPB, cardiopulmonary bypass; LIMA, left internal mammary artery; RIMA, Right internal mammary artery.

6 to 12 months after ablation, irrespective of clinical symptoms [Shah 2018]. Direct current electrocardioversion was performed in patients with postablation AF not stopped or prevented by maintenance doses of antiarrhythmic drugs, as deemed necessary by the electrophysiology team. All patients stopped oral anticoagulants 3 months after surgery.

\section{Statistical Analysis}

Data are reported as absolute and relative frequencies. The distribution of variables was analyzed by $\chi 2$ test or Fisher's exact probability test. Continuous variables that are normally distributed are presented as arithmetic means and standard deviation (SD), and those nonnormally distributed are presented as median and interquartile range (IQR). Friedman's test was used to assess change in New York Heart Association (NYHA) class from baseline. Logistic regression analysis was used to assess the factors affecting the efficacy of BRA in achieving sinus rhythm restoration and preventing postablation AF recurrence. Potential predictor variables were grouped by clinically relevant measurement (preoperative $\mathrm{AF}$ type permanent versus paroxysmal; preoperative $\mathrm{AF}$ duration $<3$ versus $>3$ years; age $<60$ versus $>60$ years). $P$ values were considered 2-tailed and not adjusted for multiple testing, and $P<.05$ indicated statistical significance. Statistical analysis was done using SPSS version 13 (SPSS Inc., Chicago, IL).

\section{RESULTS}

Baseline and demographic data for the 168 patients enrolled in the study are shown in Table 1. Group I included
97 patients $(57.7 \%)$ who underwent CABG combined with BRA for permanent AF, and group II included 71 patients (42.3\%) who underwent CABG combined with BRA for paroxysmal AF. Baseline and demographic data were compared between groups, and differences were statistically nonsignificant as shown in Table 1.

All relevant operative data are shown in Table 2. Operative values showed no significant differences between groups I and II; nor did data of completeness of revascularization $(P=$ $.8365)$. Complete revascularization of all preoperative target coronary vessels with vessel size $\geq 1.5$ and $>60 \%$ significant stenosis was achieved in 87 of 97 patients $(89.7 \%$ ) in group I and 63 of $71(88.7 \%)$ in group II. Intraoperative incomplete revascularization decision for the preoperative target coronary vessels was made when the target coronary vessel was not suitable for anastomosis or reconstruction. Ten of 97 patients $(10.3 \%)$ in group I had intraoperative incomplete revascularization and 8 of $71(11.3 \%)$ in group II, with no significant difference between groups $(P=.8365)$.

Postoperative follow-up data are described in Table 3. Group II patients had better sinus rhythm restoration rate after BRA with CABG than group I patients and a significantly lower rate for recurrent AF after BRA with CABG in comparison with group I patients $(P=.005)$. No deaths were recorded within the first 30 postoperative days in either group. During the hospital stay, 10 of 168 patients (5.9\%) had postablation recurrent AF: 6 of 168 had early successful direct current electrocardioversion, and in 4 , sinus rhythm was restored spontaneously. Six of 168 patients $(3.6 \%)$ had temporary atrioventricular block and needed temporary external pacemakers, 5 of whom converted to sinus rhythm 
Table 3. Postoperative Follow-Up Data of Patients Who Underwent AF Ablation Combined with CABG*

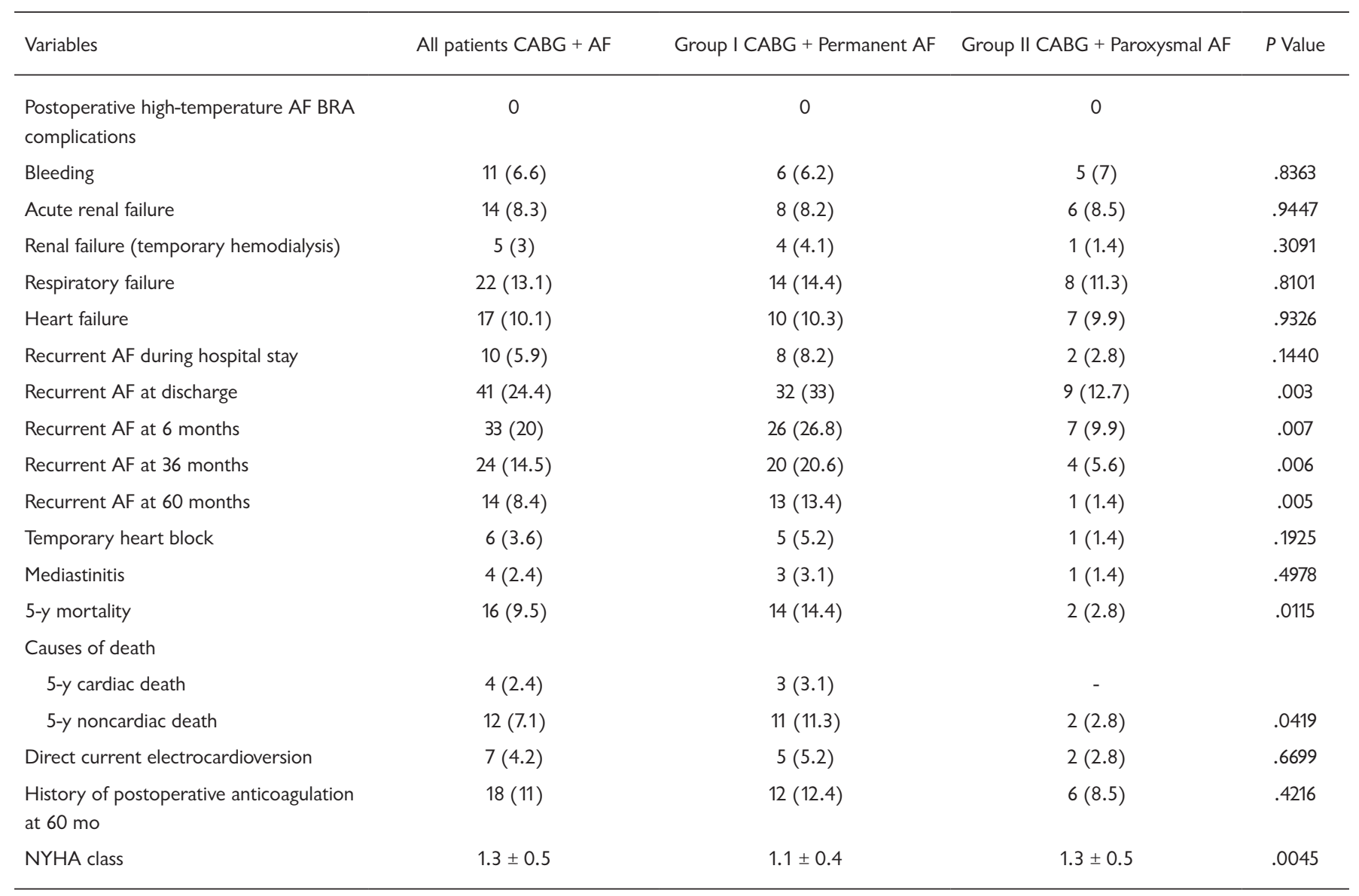

*Data are $\mathrm{n}(\%)$ or mean \pm SD.

spontaneously. The remaining patient needed permanent pacemaker implantation because of persisting bradycardia. Atrial flutter was not recorded in any of the patients. Overall mortality at 60 months was significantly lower in group II $(2[2.8 \%])$ compared with group I $(14[14.4 \%] ; P=.01)$. The 2 patients in group II died from noncardiac causes (chest infection and septicemia), 3 patients in group II died from cardiac causes (low cardiac output), and the remaining 11 patients in group II died from noncardiac causes (chest infection and septicemia). The Kaplan-Meier curve survival rate at 60 months' follow-up is presented in Figure 3. The survival rate was significantly higher in group II than in group I (94\% versus $72 \% ; P=.0003)$. The $95 \%$ confidence interval of the Cox hazards survival regression ratio was significantly different between groups (0.1792 [0.04069 to 0.7896]; $P=.006$ ).

At discharge, 127 of 168 patients $(75.6 \%)$ had sinus rhythm, and 41 of $168(24.4 \%)$ had postablation recurrent AF. The sinus rhythm restoration rate was $80.0 \%$ (133 of 166) at 6 months, $85.5 \%$ (142 of 166) at 36 months, and $91.6 \%$ (152 of 166) at 60 months. Logistic regression analysis identified preoperative $\mathrm{AF}$ type and duration as predictors of $\mathrm{AF}$ recurrence after BRA with CABG. Permanent (group 1) versus paroxysmal (group II) AF type before surgery was associated with a significantly increased risk of AF recurrence after BRA in group I patients in comparison to group II patients $(P=$ .003 at time of discharge, $P=.007$ at 6 months, $P=.006$ at 36 months, and $P=.005$ at 60 months). Preoperative AF duration $>3$ years was observed as a remarkably significant risk factor for recurrent $\mathrm{AF}$ after $\mathrm{BRA}$ with $\mathrm{CABG}$ in comparison to preoperative $\mathrm{AF}$ duration $<3$ years $(P<.0001$ at time of discharge, $P<.0001$ at 6 months, $P<.0001$ at 36 months, and $P=.0001$ at 60 months), as shown in Table 4. However, 94 of 110 patients $(85.5 \%)$ with preoperative $\mathrm{AF}$ for $<3$ years maintained sinus rhythm restoration at discharge compared with 33 of $58(57 \%)$ with preoperative $\mathrm{AF}$ for $>3$ years $(P$ $<.0001$ ), and 107 of 110 patients $(97.3 \%)$ with preoperative $\mathrm{AF}$ for $<3$ years maintained sinus rhythm restoration at 60 months' follow-up compared with 46 of 58 (79.3\%) with preoperative $\mathrm{AF}$ for $>3$ years $(P=.0001)$. At 60 months, 148 of 166 patients $(89 \%)$ did not need anticoagulation. Among those receiving oral anticoagulation were all 14 patients with post-BRA recurrent AF and 4 in whom postoperative sinus rhythm was restored at 36 months.

At 60 months, postoperative NYHA class was much improved in group II compared with group I $(P=.004)$, probably because of the higher incidence of postoperative 


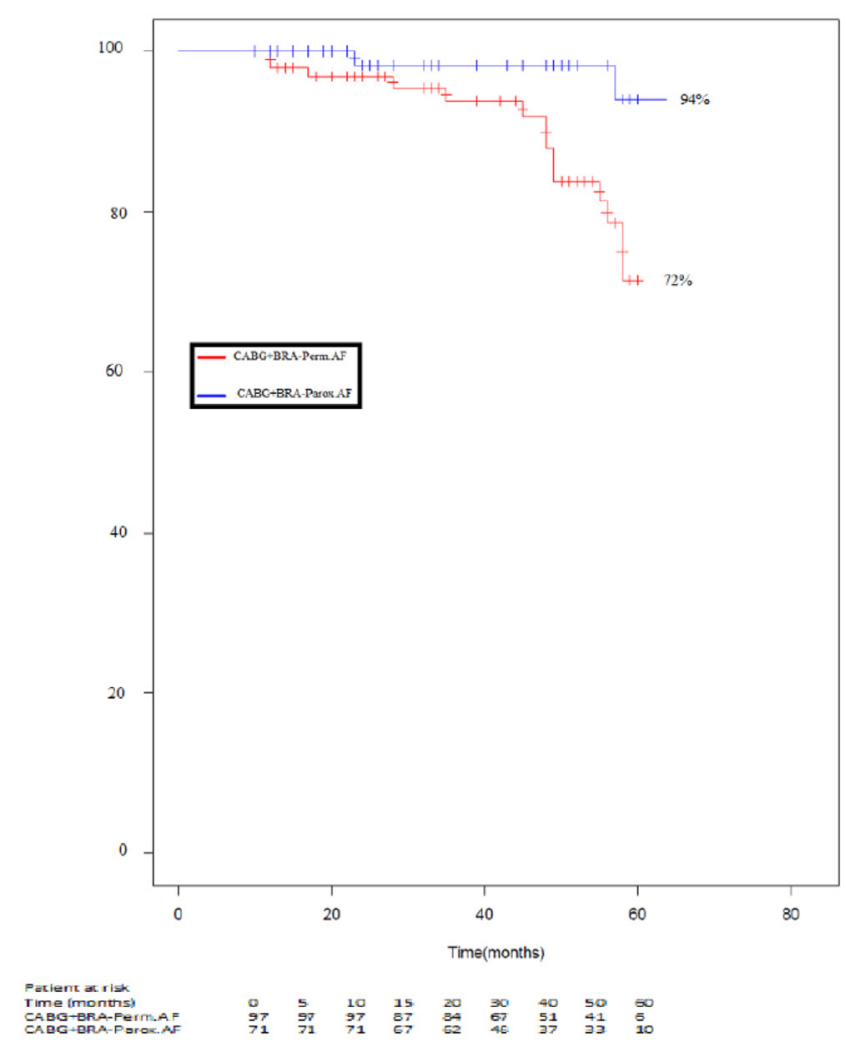

Figure 3. Kaplan-Meier curves: survival rate at 60 months of followup in patients who underwent $A F$ ablation combined with $C A B G$.

$\mathrm{AF}$ recurrence in group I patients in comparison to group II patients. Mean NYHA class had significantly decreased after BRA combined with CABG, from $2.7 \pm 0.6$ preoperatively to $1.3 \pm 0.5$ postoperatively $(P<.0001)$. Among the patients with sinus rhythm restoration, 114 of 168 were NYHA I and 38 of 168 NYHA II, whereas among those with post-BRA recurrent AF, only 2 patients were NYHA I and 12 were NYHA II. Neither age $(P=.15, .12, .11$, and .10 at discharge, 6,36 , and 60 months, respectively) nor sex $(P=.72, .51, .29$, and .09 ) had a predictive effect on sinus rhythm restoration after BRA with CABG.

Complications are shown in Table 3. No severe BRA complications (e.g., damage of mediastinal structures, esophageal or tracheal perforation) were reported in our study. Acute renal failure occurred in $14(8.3 \%)$ of 168 patients; only 5 needed temporary hemodialysis.

\section{DISCUSSION}

In this prospective study, we demonstrated that among $\mathrm{AF}$ patients undergoing combined surgical ablation and CABG, BRA achieves high rates of postoperative sinus rhythm restoration. Preoperative AF type and duration influenced the probability of establishing stable sinus rhythm postoperatively. We found that preoperative AF type and AF duration
Table 4. Influence of Preoperative AF Duration on Postoperative AF Recurrence Rate in Patients Who Underwent AF Ablation Combined with CABG*

\begin{tabular}{lccc}
\hline Time & $\begin{array}{c}\text { Recurrent AF in CABG + BRA } \\
\text { Preoperative AF }<3 \text { years }\end{array}$ & $\begin{array}{c}\text { Preoperative } \\
\text { AF }>3 \text { years }\end{array}$ & $P$ Value \\
\hline$N$ & $110(65.5)$ & $58(43.5)$ & \\
$\begin{array}{l}\text { At time of } \\
\text { discharge }\end{array}$ & $16(14.5)$ & $25(43)$ & $<.0001$ \\
At 6 mo & $10(9.1)$ & $23(39.7)$ & $<.0001$ \\
At 36 mo & $6(4.5)$ & $18(30)$ & $<.0001$ \\
At 60 mo & $3(2.7)$ & $11(20.7)$ & .0001 \\
\hline
\end{tabular}

*Data are $n(\%)$.

are not independent risk factors for BRA failure, but that myocardial electrophysiological damage correlates with $\mathrm{AF}$ type and duration. Our study presents a successful approach of $\mathrm{AF}$ treatment combined with $\mathrm{CABG}$ in cardiac patients and will likely stimulate further research on the topics of $\mathrm{AF}$ ablation combined with CABG to improve clinical outcomes. The results of our study are sufficiently innovative and will likely advance the research field of management of AF in cardiac patients.

Our institution started using BRA combined with CABG to decrease the complications of ablation invasiveness, ischemic time, and risk of bleeding. However, the approach is still incompletely understood. During the 2000s, BRA technology, which creates scars without cutting the tissue, renewed interest in combining surgical $\mathrm{AF}$ ablation with CABG. Geidel et al. [2006] demonstrated that BRA technology combined with CABG for permanent AF management is as safe as mitral valve replacement. BRA has a significantly shorter ablation procedure time than other technologies [Geidel 2006].

Improved cardiac output and survival improvement are the major clinical advantages of sinus rhythm restoration in the management of AF combined with CABG [Gillinov 2005]. The success of BRA needs to be evaluated not only for sinus rhythm restoration achievement over time, but also for the improvement in the quality of life in cardiac patients with $\mathrm{AF}$ [Canale 2018]. BRA has the advantage of an auditory feedback signal from the console system, so the surgeon is able to assess whether the lesion has reached the entire thickness of the atrial wall [Canale 2018; Vural 2018].

Different factors affect the effectiveness of radiofrequency ablation, including source of energy, ablation line sets, and type of cardiac surgery [Sie et al. 2004]. Sinus rhythm was restored in $91.6 \%$ (152 of 166 patients) at 60 months. More than $40 \%$ of patients had paroxysmal AF, and nearly $60 \%$ had permanent AF preoperatively. Sinus rhythm restoration in the early postoperative period was greater among patients with paroxysmal $\mathrm{AF}$ (group II) than those with permanent $\mathrm{AF}$ (group I), with a remarkably significant difference between the groups on long-term clinical follow-up. 
Despite advances in BRA technology and technique, recurrent $\mathrm{AF}$ may occur. However, early $\mathrm{AF}$ recurrence after BRA does not necessarily indicate long-term failure and should be managed with direct current electrocardioversion with or without antiarrhythmic drugs. Our results, in agreement with those of Sie et al. [2004], indicate that when ablation line sets are made twice at the same site, it would be safe and enough to avoid a gap in the ablation line. This is supported by the high sinus rhythm restoration rate in this study. Our study demonstrates the superiority of BRA in terms of clinical outcomes and increased frequency of sinus rhythm restoration, attributable to its complete efficient transmural ablation lines. In other words, advances in technology and techniques have made surgical intervention for $\mathrm{AF}$ easier, and surgeons have embraced it.

\section{Limitations}

The study is limited by a relative small sample size, nonrandomized clinical trial, and homogeneity in terms of the type of cardiac surgery, but it meets the necessary requirements on the basis of our primary key objectives in sinus rhythm restoration.

\section{Conclusions}

Our study assessed the clinical outcomes of BRA conducted together with CABG in AF patients. A sinus rhythm restoration rate of almost $91.6 \%$ can be reached after 5 years of follow-up. Preoperative AF type and duration can predict the success of BRA combined with CABG; specifically, preoperative permanent $\mathrm{AF}$ of long duration increases the risk of BRA failure.

\section{REFERENCES}

Benussi S, Pappone C, Nascimbene S, Oreto G, Caldarola A, Stefano PL, et al. A simple way to treat chronic atrial fibrillation during mitral valve surgery: The epicardial radiofrequency approach. Eur J Cardiothorac Surg 2000;17:524-529.

Cameron A, Schwartz MJ, Kronmal RA, Kosinski AS. Prevalence and significance of atrial fibrillation in coronary artery disease (CASS Registry). Am J Cardiol 1988;61:714-717.

Canale L, Bruno Azevedo B, Marcelo Correia M, et al. Bipolar versus unipolar energy in the surgical ablation of atrial fibrillation in patients with mitral valve surgery. Heart Vessels Transplant 2018;4:106-122.

Fuster V, Ryden LE, Cannom DS, et al. ACC/AHA/ESC 2006 guidelines for the management of patients with atrial fibrillation: A report of the American College of Cardiology/American Heart Association Task Force on Practice Guidelines and the European Society of Cardiology Committee for Practice Guidelines (Writing Committee to Revise the 2001 Guidelines for the Management of Patients with Atrial Fibrillation): Developed in collaboration with the European Heart Rhythm Association and the Heart Rhythm Society. Circulation 2006;114:e257-e354.

Gammie JS, Haddad M, Milford-Beland S, et al. Atrial fibrillation correction surgery: Lessons from the Society of Thoracic Surgeons National Cardiac Database. Ann Thorac Surg 2008;85:909-914.

Geidel S, Ostermeyer J, Lab M, et al. Permanent atrial fibrillation ablation surgery in CABG and aortic valve patients is at least as effective as in mitral valve disease. Thorac Cardiovasc Surg 2006;54:91-95.

Gillinov AM, McCarthy PM, Blackstone EH, et al. Surgical ablation of atrial fibrillation with bipolar radiofrequency as the primary modality. J Thorac Cardiovasc Surg 2005;129:1321-1328.

Haissaguerre M, Jais P, Shah DC, Takahashi A, Hocini M, Quiniou G, et al. Spontaneous initiation of atrial fibrillation by ectopic beats originating from the pulmonary veins. N Engl J Med 1998;339:659-666.

Martin-Suarez S, Claysset B, Botta L, et al. Surgery for atrial fibrillation with radiofrequency ablation: Four years experience. Interact Cardiovasc Thorac Surg 2007;6:71-76.

Melby JS, Schuessler RB, Damiano RJ Jr. Ablation technology for the surgical treatment of atrial fibrillation. ASAIO J 2013;59:461-468.

Melby SJ, Lee AM, Zierer A, Boineau JP, Schuessler RB, Damiano J. Do surgical ablations have to be transmural to prevent the propagation of atrial fibrillation? J Am Coll Surg 2005;201:S23.

Melo J, Adragao P, Neves J, et al. Endocardial and epicardial radiofrequency ablation in the treatment of atrial fibrillation with a new intraoperative device. Eur J Cardiothorac Surg 2000;18:182-186.

Miyasaka Y, Barnes ME, Gersh BJ, et al. Secular trends in incidence of atrial fibrillation in Olmsted County, Minnesota, 1980 to 2000 and implications on the projections for future prevalence. Circulation 2006;114:119-125.

Otterstad JE, Kirwan BA, Lubsen J, et al. Action investigators. Incidence and outcome of atrial fibrillation in stable symptomatic coronary disease. Scand Cardiovasc J 2006;40:152-159.

Prasad SM, Maniar HS, Camillo CJ, et al. The Cox Maze III procedure for atrial fibrillation: Long-term efficacy in patients undergoing lone versus concomitant procedures. J Thorac Cardiovasc Surg 2003;126:1822-1827.

Prasad SM, Maniar HS, Diodato MD, Schuessler RB, Damiano J. Physiological consequences of bipolar radiofrequency energy on the atria and pulmonary veins: A chronic animal study. Ann Thorac Surg 2003;76:836-842.

Santangeli P, Di Biase L, Natale A. Ablation versus drugs: What is the best first-line therapy for paroxysmal atrial fibrillation? Antiarrhythmic drugs are outmoded and catheter ablation should be the first-line option for all patients with paroxysmal atrial fibrillation: Pro. Circ Arrhyth Electrophysiol 2014;7:739-746.

Shah S, Barakat AF, Saliba WI, Rehman KA, Tarakji KG, Rickard J, et al. Recurrent atrial fibrillation after initial long-term ablation success. Circ Arrhythm Electrophysiol 2018;11:e005785.

Sie HT, Beukema WP, Elvan A, Ramdat Misier AR. Long-term results of irrigated radiofrequency modified maze procedure in 200 patients with concomitant cardiac surgery: Six years experience. Ann Thorac Surg 2004;77:512-517.

Starck C, Steffel J, Holubec T, Falk V. Current aspects of atrial fibrillation surgery. Cardiovasc Med 2015;18:181-185.

Vural U, Balcı AY, Ağlar AA, Kızılay M. Which method to use for surgical ablation of atrial fibrillation performed concomitantly with mitral valve surgery: Radiofrequency ablation versus cryoablation. Braz J Cardiovasc Surg 2018;33:542-552

Wyse DG, Waldo AL, DiMarco JP, et al. A comparison of rate control and rhythm control in patients with atrial fibrillation. N Engl J Med 2002;347:1825-1833. 